\title{
Profiling single-molecule reaction kinetics under nanopore confinement
}

\author{
Wei Liu ${ }^{1}$, Zhong-Lin Yang ${ }^{1}$, Chao-Nan Yang ${ }^{1}$, Yi-Lun Ying ${ }^{1} 2^{*}$, Yi-Tao Long ${ }^{1}$
}

\begin{abstract}
Affiliations
1 State Key Laboratory of Analytical Chemistry for Life Science, School of Chemistry and Chemical Engineering, Nanjing University, Nanjing, 210023, P.R. China.

${ }^{2}$ Chemistry and Biomedicine Innovation Center, Nanjing University, Nanjing, 210023, P.R. China
\end{abstract}

\section{Email: yilunying@nju.edu.cn}

\section{Keywords}

Single Molecule Reaction Kinetics • Peptide Reaction• Aerolysin Nanopore - Confined Space• Thiol-Based Chemistry

\begin{abstract}
The development of single-molecule reaction inside nano-confinement is benefit to study the intrinsic molecular mechanism of a complex chemical reaction. However, the reaction kinetics model of single-molecule reaction inside confinement remains elusive. Herein we engineered the Aerolysin nanopore reactor to elaborate the single-molecule reaction kinetics inside nano-confinement. By identifying bond forming and non-forming events directly, a four-state kinetics model is proposed for the first time. Our results demonstrated that the single-molecule reaction kinetics inside a nanopore depends on the voltagedependent frequency of captured individual reactant and the fraction of effective collision inside nanopore confined space. This new insight will guide the design of nanoconfinement for resolving the single-molecule chemistry, and shed light on the mechanistic understanding of dynamic covalent chemistry in-side a nanopore.
\end{abstract}

In nature, nanoconfinement is the key feature for the enzymatic reaction, where the substrates selectively encounter with the reactive site $^{1}$. The confined intermolecular interactions between enzymes and substrates facilitate pre-organization of reagents, giving exceptional reaction efficiency. Inspired by enzyme confinement, reaction vessels at micro/nanoscale were engineered to capture and organize the reagent, which are formed by nanoparticles ${ }^{2}$, micelles ${ }^{3}$, supramolecular cave ${ }^{4}$, coordination cages ${ }^{5}$ and proteins ${ }^{6-8}$. In this way, the confined space is further designed to isolate single reactant from the bulk, which benefits to study the reactive intermediates and the reaction kinetics.

Biological nanopores utilize membrane proteins to form nanoconfinement for accommodating single molecule ${ }^{9-13}$. The reactive groups could be spatially designed alone the polypeptide chain of the protein nanopore. Under bias voltage, the single reactant is confined inside a nanopore in time and space with the controllable movements and direction. The covalent bond formation can be triggered at a specific reactive site, that regulates the ionic current through a nanopore ${ }^{14,15}$. Coupled with high bandwidth current recording system, nanopore could real-time report reactive intermediates ${ }^{16-18}$, study the 
reaction kinetics ${ }^{19-21}$ and explore reaction trajectory ${ }^{22-24}$ at single-molecule level.

Generally, the reaction between single reactant molecule and the reactive site inside nanopore is proposed as pseudo first order process and the reaction rate is calculated by concentration-dependent experiment ${ }^{25}$. However, the capture and driving force of the reactant need to be considered inside nanopore confinement. For example, does the driven force of the reactant affect the reaction kinetics? How does the capture frequency regulate the reactive kinetics? In enzyme-catalysis process, the reaction kinetics is divided into substrate binding and product conversing which is supported by Michaelis-Menten equation. Inspired by the enzyme-catalysis kinetics theory ${ }^{26}$, herein we classify the singlemolecule reaction process inside nanopore into three steps which are 1) capture of single reactant, 2) trigger of single-molecule reaction at specific site and 3) possible dissociation of the single reactant from the reactive site inside nanopore. To profile kinetics of this reaction, the rapid translocation of the single reactant should be slow down for clearly recording the above three processes. Herein we designed series of thiol-containing peptides $\left(\boldsymbol{R}_{\mathbf{1}}-\boldsymbol{R}_{\mathbf{5}}\right)$ as reactants to bond with cysteine mutant Aerolysin nanopore (AeL). Both the reaction and non-reaction process produce the distinguishable ionic current signatures. This model system ensures us to study the single-molecule reaction kinetics. Our results demonstrated that the reaction kinetics inside a nanopore depends on reactant capture rate and fraction of effective collision, which could further be described as a four-state kinetic model.

The disulfide formatting and breaking reaction was built inside an AeL nanopore which contains seven inward-facing cysteine at position 238 on the seven subunits (Figure 1a). The conductance of K238C AeL is $(5.2 \pm 0.2) \times 10^{-4} \mathrm{~S}(\mathrm{n}=5)$ at $20.0 \pm 2.0^{\circ} \mathrm{C}$ (Figure S12). The thiol derivate, thiol-containing peptide $(\boldsymbol{R})$, is designed and contains Glutamic acids (E) segment as guiding sequence at $\mathrm{N}$-terminal and a reactive cysteine at $\mathrm{C}$-terminal. We use $\boldsymbol{R}_{\mathbf{1}}$ (EEESGSGSGSGSGSC) as a model reactant to demonstrate our proposed kinetics model. The ionic events of $\boldsymbol{R}_{\mathbf{1}}$ could be classified into Type I of reaction event with long durations (> $100 \mathrm{~ms}$ ) and Type II of non-reaction event with short durations (Figure 1 and Figure S3-S5, details see in Supplementary Information 2.2).
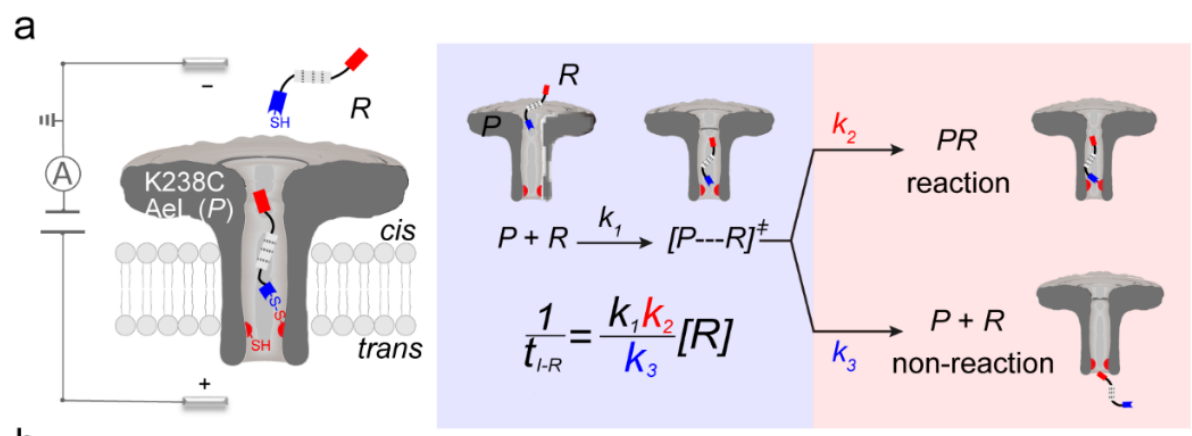

b

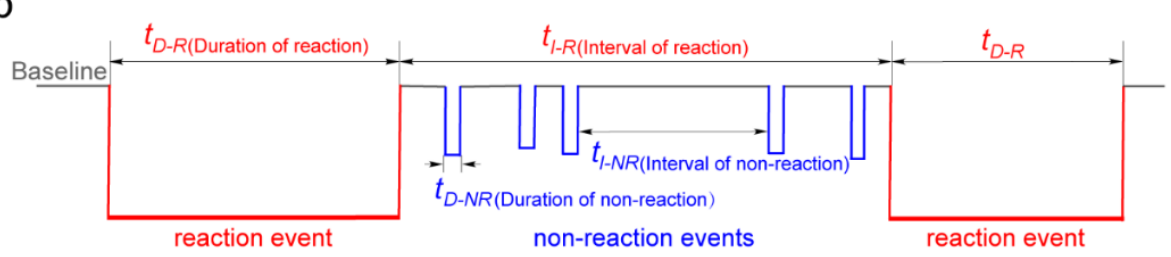


Figure 1. (a) A schematic illustration of single-molecule reaction between $\boldsymbol{R}$ and K238C AeL nanopore $(\boldsymbol{P})$; (b) The current trace model for the reaction event and non-reaction event. The kinetics model describes the four state of: 1 ) capturing of $\boldsymbol{R}$ into the nanopore, 2) energizing a cysteine residue for $[\boldsymbol{P}---\boldsymbol{R}]^{\ddagger}$ by the collision of $\boldsymbol{R}$ toward the K238C site, 3) forming a covalent bond between $\boldsymbol{R}$ and K238C; and 4) releasing of $\boldsymbol{R}$ after a deactivation. The $k_{1}, k_{2}$ and $k_{3}$ represents the kinetics constant of each step. [R] refers to the concentration of reactant. $t_{I-R}$ stands for the interval time between adjacent reaction events. $t_{I-N R}$ refers to the interval time between adjacent non-reaction events. $t_{D-R}$ denotes the duration time of reaction events. $t_{D-N R}$ assigns the duration of non-reaction events.

Note that the reaction and non-reaction events were observed under whole bias voltage ranging from $+60 \mathrm{mV}$ to $+110 \mathrm{mV}$ (with respect to the cis chamber). These results reveal that the single reactant could undergo either reaction or non-reaction (but translocation) pathway as it enters the nanopore. Therefore, we proposed a four-state kinetic model to describe single-molecule reactions inside a nanopore as shown in Figure 1a and eq. 1-3 as follows. When the reactant peptide $(\boldsymbol{R})$ confines into the $\mathrm{K} 238 \mathrm{C} \mathrm{AeL}(\boldsymbol{P})$ from the cis side, it continuously moves and collides along the inner surface of K238C AeL under the bias voltage. Then, the $R$ reaches the reactive thiol groups at $\mathrm{K} 238 \mathrm{C}$ site, and reacts with the cysteine residue, giving an intermediate state of $[\boldsymbol{P}---\boldsymbol{R}]^{\ddagger}$ (eq. 1 ), similar to enzymesubstrate binding. Due to the confinement effect, the $[\boldsymbol{P}---\boldsymbol{R}]^{\ddagger}$ acquires sufficient energy to enable the covalent bond formation, resulting in a disulfide product of $\boldsymbol{P R}$ (eq. 2). This process of $\boldsymbol{P}+\boldsymbol{R} \rightarrow[\boldsymbol{P}---\boldsymbol{R}]^{\ddagger} \longrightarrow \boldsymbol{P} \boldsymbol{R}$ yields reaction events. Alternatively, the $[\boldsymbol{P}---\boldsymbol{R}]^{\ddagger}$ would be dissociated in which case the $\boldsymbol{R}$ escapes from trans side of the nanopore after a rapid interaction but not bind-forming with K238C site (eq. 3). This second pathway of $\boldsymbol{P}+\boldsymbol{R} \rightarrow[\boldsymbol{P}$ $-\boldsymbol{R}]^{\ddagger} \longrightarrow \boldsymbol{P}+\boldsymbol{R}$ generates non-reaction events.

$$
\begin{aligned}
& \boldsymbol{P}+\boldsymbol{R} \stackrel{k_{1}}{\longrightarrow}[\boldsymbol{P}--\boldsymbol{R}]^{\ddagger} \\
& {[\boldsymbol{P}--\boldsymbol{R}]^{\ddagger} \stackrel{k_{2}}{\longrightarrow} \boldsymbol{P R}} \\
& {[\boldsymbol{P}--\boldsymbol{R}]^{\ddagger} \stackrel{k_{3}}{\longrightarrow} \boldsymbol{P}+\boldsymbol{R}}
\end{aligned}
$$

wherein the rate constant of $k_{1}$ ascribes for the reactant capture of eq. 1 , the rate constant $k_{2}$ for product conversion of eq. 2 and the rate constant $k_{3}$ for the deactivation reaction of $[\boldsymbol{P}---\boldsymbol{R}]^{\ddagger}$ of eq. 3. As described in a previous study ${ }^{27}$, the reactant captured process inside a nanopore follows a pseudo first order process. The $k_{1}$ could be estimated by eq. 4 :

$$
k_{1}=\frac{1}{t_{I}[R]}=\frac{f_{[P \cdots R]^{\ddagger}}}{[R]} \approx \frac{f_{P+R}}{[R]}=\frac{1}{t_{I-N R}[R]}
$$

whereas $t_{I}$ refers to the interval time of all events (both reaction and non-reaction events), and $t_{I-N R}$ denotes to the interval time of non-reaction events. $[R]$ is the concentration of reactant $\boldsymbol{R} . f_{[\boldsymbol{P}+\boldsymbol{R}]^{\ddagger}}$ is the frequency of all captured events and $f_{\boldsymbol{P}+\boldsymbol{R}}$ refers to the frequency of non-reaction event. Since the number of non-reaction events is much larger than that of reaction events in our cases, $f_{[\boldsymbol{P}+\boldsymbol{R}]^{\ddagger}}$ is approximated to $f_{\boldsymbol{P}+\boldsymbol{R}}$. In order to understand the single-molecule reaction kinetics when the reactant was captured, we use 
the effective collision fraction (ECF) ${ }^{28}$ for describing the disulfide bond formation possibility inside a $\mathrm{K} 238 \mathrm{C}$ AeL as shown in eq. 5 :

$$
E C F=\frac{N_{P R}}{N} \approx \frac{N_{P R}}{N_{P+R}}=\frac{f_{P R}}{f_{P+R}}=\frac{t_{I-N R}}{t_{I-R}} \times 100 \%
$$

whereas refers to the number of all capture of $\boldsymbol{R}, N_{\boldsymbol{P R}}$ assigns to the number of reaction events, and $N_{P+R}$ defines to the number of non-reaction events. Accordingly, $f_{P R}$ is the frequency of product $\boldsymbol{P R}$, that is, the reaction rate. The ECF can be calculated from the interval time between two adjacent reaction events $\left(t_{I-R}\right)$ and two adjacent non-reaction events $\left(t_{I-R}\right)$. Subsequently, the reaction rate $f_{P R}$ can be described by eq. 6 :

$$
f_{P R}=\frac{1}{t_{I-R}}=E C F \times f_{P-R}=k_{1} E C F[R]
$$

According to eq. 6 , the kinetics of single-molecule reaction inside nanopore confinement could be regarded as a pseudo first order process when $f_{P+R}$ is much larger than $f_{P R}$. To verify the proposed single-molecule kinetics model, we analysis the reaction event between $\boldsymbol{R}_{\mathbf{1}}$ and $\mathrm{K} 238 \mathrm{C}$ AeL under various concentrations. As expected, the ECF gives a constant value of $(17.0 \pm 2.0) \%$ while $f_{P R}$ is linearly increased with the $\left[\boldsymbol{R}_{\mathbf{1}}\right]$ concentration (Figure 2a). These results demonstrate that ECF is nearly irrelevant to the $\left[\boldsymbol{R}_{\mathbf{1}}\right]$ concentration. The high concentration of $\left[\boldsymbol{R}_{\mathbf{1}}\right]$ just ensures the effective trapping and formation of $\left[\boldsymbol{P}---\boldsymbol{R}_{\mathbf{1}}\right]^{\ddagger}$, leading to a high occurrence of reaction events.
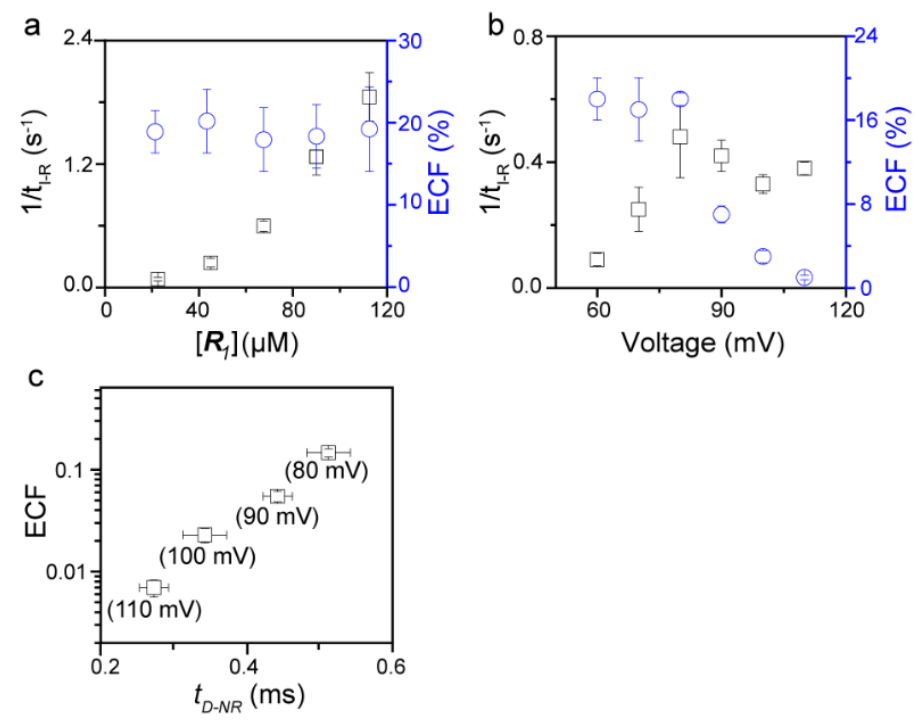

Figure 2. Kinetics evaluation of $\boldsymbol{R}_{\mathbf{1}}$ reacting with K238C AeL nanopore. (a) The reaction rate $\left(f_{\boldsymbol{P}}\right)$ and effective collision fraction (ECF) under different $\boldsymbol{R}_{\boldsymbol{1}}$ concentration. (b) Reaction rate $\left(f_{P R}\right)$ and ECF under different voltage from $+60 \mathrm{mV}$ to $+110 \mathrm{mV}$. (c) The relationship between ECF and non-reaction event duration $\left(t_{D-N R}\right)$. The voltage dependence results of $t_{D-N R}$ is shown in Figure S5a.

The results from voltage dependent studies show that the higher voltage provides a larger $k_{1}$ due to the stronger driving force for the negatively charge $\boldsymbol{R}_{\boldsymbol{1}}$ (Figure S4b). Consequently, $f_{P R}$ gradually increases from $+60 \mathrm{mV}$ to $+80 \mathrm{mV}$ (Figure $2 \mathrm{~b}$ ). However, $f_{\boldsymbol{P} R}$ does not show significantly change at bias voltage from $+80 \mathrm{mV}$ to $+110 \mathrm{mV}$. When the bias voltage increased from $+60 \mathrm{mV}$ to $+80 \mathrm{mV}$, the ECF showed a constant value of $(18.0 \pm 2.0) \%$ 
(Figure $2 \mathrm{~b}$ ). Then, it decreased rapidly as the bias voltage exceeds the threshold of +80 $\mathrm{mV}$. The low bias voltage $(<+80 \mathrm{mV})$ is in favor of the formation pathway of disulfide bond. However, the high bias voltage $(>+80 \mathrm{mV})$ is prone to deactivation of $\left[\boldsymbol{P}_{---\boldsymbol{R}_{1}}\right]^{\ddagger}$ to release the $\boldsymbol{R}_{\mathbf{1}}$ from the trans side. At $+110 \mathrm{mV}, f_{\boldsymbol{P}+\boldsymbol{R}}$ is over 100 times larger than $f_{\boldsymbol{P} \boldsymbol{R}}$, which suggests that most of the $\boldsymbol{R}_{\mathbf{1}}$ molecules undergo ineffective bonding at the high bias voltage (Table S1). More interestingly, at low voltage $(<+80 \mathrm{mV})$, the $f_{P R}$ is increased from +60 $\mathrm{mV}$ to $+80 \mathrm{mV}$, which is determined by the process of eq. 1 . At high voltage $(>+80 \mathrm{mV})$, the destabilization of $\left[\boldsymbol{P}---\boldsymbol{R}_{1}\right]^{\ddagger}$ significantly shifts the reaction pathway to non-reaction pathway, thereby inhibiting the eq. 2 process. Process of eq. 3 becomes the rate-limited step for the single-molecule reaction. The reaction kinetics is dominated by the bonding efficiency between $\boldsymbol{R}_{\mathbf{1}}$ and thiols group at 238 site rather than the bulk concentration of $\boldsymbol{R}_{\mathbf{1}}$. Thus, the ECF can be expressed by the rate constant of reaction triggered by eq. $2\left(k_{2}\right)$ and deactivation reaction of $[\boldsymbol{P}---\boldsymbol{R}]^{\ddagger}$ in eq. $3\left(k_{3}\right)$ as follows:

$$
E C F=\frac{k_{2}}{k_{3}} \times 100 \%
$$

Our previous studies demonstrated that 238 site locates at energy barrier for the singlemolecule translocation ${ }^{29}$. Compare with WT AeL, K238C prolongs the duration of poly $(\mathrm{dA})_{4}$ for about 7 times $^{30}$. Therefore, we assumed the $k_{3}$ is correlation to the translocation velocity of $\boldsymbol{R}_{\boldsymbol{1}}$ through K238C AeL. The linear relationship between $\lg (\mathrm{ECF})$ and duration time of non-reaction event $\left(t_{D-N R}\right)$ further support our hypothesis (Figure $2 \mathrm{c}$ ). The increasing residence time of a single reactant at the reaction site can effectively improve reaction efficiency, which provides a basis for the design of nanopore and reactant for singlemolecule synthesis.

To further confirm our kinetics model, we designed a series of cysteine contained peptides. As shown in Figure $3 \mathrm{a}-\mathrm{b}$, two thiol-containing peptides, $\boldsymbol{R}_{\mathbf{2}}$ and $\boldsymbol{R}_{\mathbf{3}}$, were designed with same length and net charges. However, their captured possibility by $\mathrm{K} 238 \mathrm{C}$ AeL are different due to the uniform distribution of charged amino acids. Similar to $\boldsymbol{R}_{\boldsymbol{1}}$, two types of events with distinguishable duration difference are classified (Figure S6a-b). Our results show that the $f_{\boldsymbol{P} \boldsymbol{R}}$ of $\boldsymbol{R}_{\mathbf{2}}$ is larger than that of $\boldsymbol{R}_{\mathbf{3}}$ (Figure $\left.3 a-b\right)$ ), while the ECF of $\boldsymbol{R}_{\mathbf{2}}$ and $\boldsymbol{R}_{\mathbf{3}}$ shows the comparable value under low voltage $(<+80 \mathrm{mV})$. These results are consistent with that of $\boldsymbol{R}_{\mathbf{1}}$, confirming that the reaction rate is captured-limitation under low voltage (< $+80 \mathrm{mV}$ ) (Figure S7a-b, Table S1). Another two peptides, $\boldsymbol{R}_{\boldsymbol{4}}$ and $\boldsymbol{R}_{\mathbf{5}}$ (Figure 3c-d) was analyzed, which remove the negative charge of guiding segments for changing their translocation rates. As expected, the order of ECF is $\boldsymbol{R}_{5}>\boldsymbol{R}_{\mathbf{4}}$ under the lower voltages ( $\leq$ $+80 \mathrm{mV}$ ). The driving force of $\boldsymbol{R}_{\mathbf{5}}$ is dominated by the electroosmotic flow, leading to slow translocation speed of $\boldsymbol{R}_{\mathbf{5}}$. (Figure S7c-d, Table S1). For all the four reactants peptides, the $f_{P R}$ is independent on voltage, however the voltage-dependent trends of ECF is consistent with $\boldsymbol{R}_{\mathbf{1}}$. Results confirm that the ECF is decreased with enhanced translocation speed of non-reaction event at high voltages $(>+80 \mathrm{mV}$ ) (Figure S8a-d). 
a
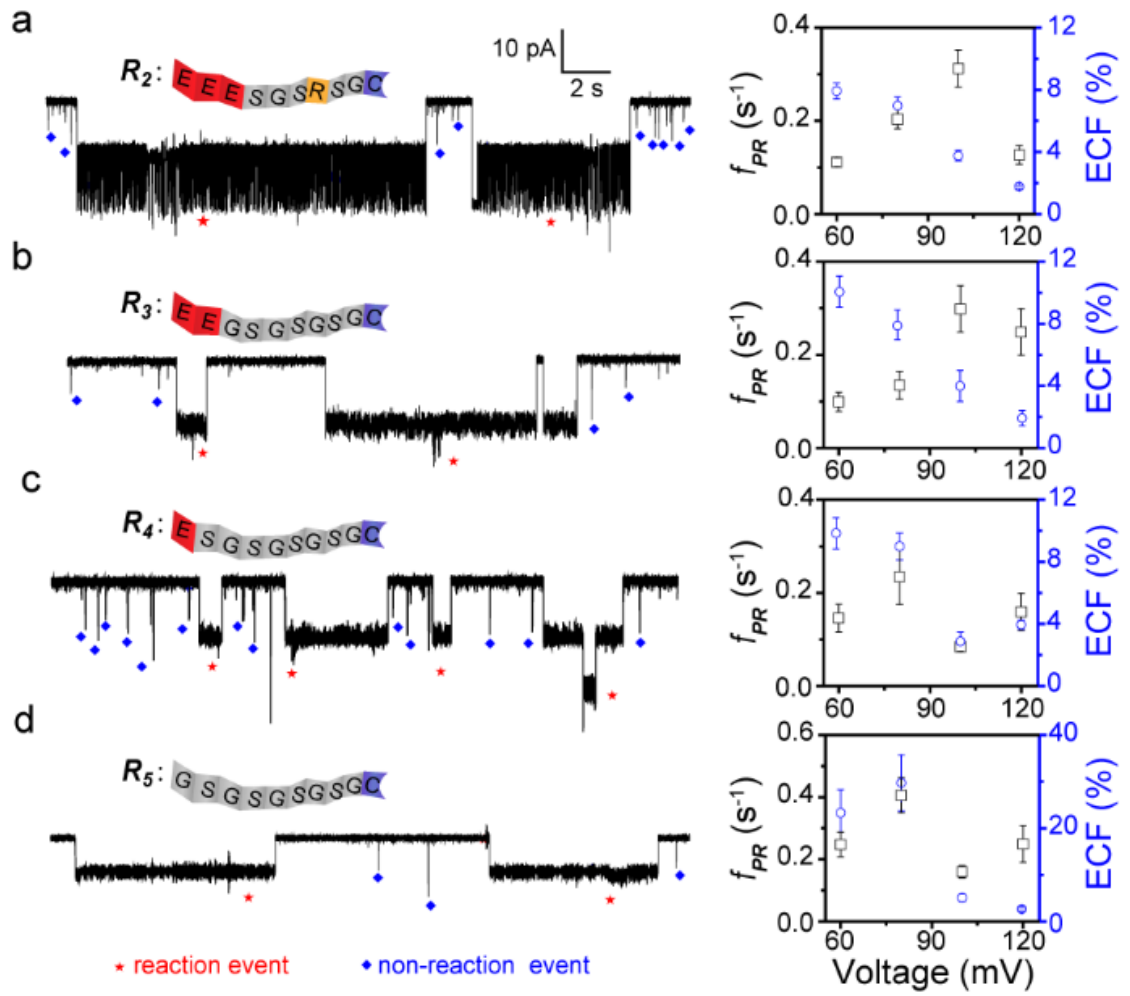

Figure 3. The single-molecule reaction of $\boldsymbol{R}_{\mathbf{2}}(\mathrm{a}), \boldsymbol{R}_{\mathbf{3}}(\mathrm{b}), \boldsymbol{R}_{\mathbf{4}}(\mathrm{c})$, and $\boldsymbol{R}_{\mathbf{5}}(\mathrm{d})$ with a K238C AeL nanopore. Left: The ionic current trace at $+60 \mathrm{mV}$. The red star and blue star represent the reaction events, and non-reaction events, respectivly. Right: voltage dependence of the reaction rate $\left(f_{P R}\right)$ and ECF. The current fluctuation of the reaction events may be attribute to the conformational changes of the peptide or the possible intermediates. All data were acquired at $20.0 \pm 2.0^{\circ} \mathrm{C}$ in $1.0 \mathrm{M} \mathrm{KCl}, 10.0 \mathrm{mM}$ Tris, and $1.0 \mathrm{mM}$ EDTA solution buffered at $\mathrm{pH} 8.0$ in the presence of $50.0 \mu \mathrm{M}$ reactant.

We surprisingly found that all reaction events could return to the initial open-pore state, illustrating that the disulfide bond could be cleaved without additional reducing agents. In order to analyze the single bond breaking process, we conducted a three-state kinetics model for describing the disulfide bond breaking under nanopore confinement (Figure S9, details see in Supplementary Information 2.5). The results show that the breaking of disulfide bond between $\boldsymbol{R}_{\mathbf{4}}$ and $\mathrm{K} 238 \mathrm{C}$ is more tolerate on the bias voltage than other four peptides.

In conclusion, the nanopore confined effect provokes "four-state kinetics module" for single-molecule reaction. The kinetics of bond forming is decided by both the possibility of captured molecule $\left(k_{1}[R]\right)$ and fraction of effective collision reaction (ECF) inside a nanopore. According to concentrations and bias voltages dependence experiments, ECF is dominated by the bond active rate constant $k_{2}$ and deactivation rate constant of $[\boldsymbol{P}---\boldsymbol{R}]^{\ddagger}$ $\left(k_{3}\right)$, which is not considered in previous nanopore researches. This novel reaction kinetics model provides a new basis for the design of nanopore reactors. We anticipant that this model could be extended to a wide range of organic and inorganic nanoconfinement for 
promoting the single-molecule chemistry.

\section{References}

(1) Grommet, A B.; Feller, M.; Klajn R. Chemical Reactivity under Nanoconfinement. Nat. Nanotech. 2020, 15, 256-271.

(2) Zdobinsky, T.; Maiti, P S.; Klajn, R. Support Curvature and Conformational Freedom Control Chemical Reactivity of Immobilized Species. J. Am. Chem. Soc. 2014, 136, 27112714.

(3) Lee, J.; Samanta, D.; Nam, H.; Zare, R. Micrometer-Sized Water Droplets induce Spontaneous Reduction. J. Am. Chem. Soc. 2019, 141, 10585-10589.

(4) Palma, A.; Artelsmair, M.; Scherman, O A. et al. Cucurbit[7]uril as a Supramolecular Artificial Enzyme for Diels-Alder Reactions. Angew. Chem. Int. Ed. 2017, 129, 1589415898.

(5) Takezawa, H.; Shitozawa, K.; Fujita, M. Enhanced Reactivity of Twisted Amides inside a Molecular Cage Nat. Chem. 2020, 12, 574-578.

(6) Dutta, S.; Whicher, J R.; Hansen, D A. et al. Structure of a Modular Polyketide Synthase. Nature 2014, 510, 512-517.

(7) Mindell, J A.; Zhan, H J.; Huynh, P D.; Collier, R J; Finkelstein, A. P. Reaction of Diphtheria Toxin Channels with Sulfhydryl-Specific Reagents: Observation of Chemical Reactions at the Single Molecule Level. P. Natl. Acad. Sci. USA 1994, 91, 5272-5276.

(8) Qing, Y.; Tamagaki-Asahina, H.; Ionescu, S A.; Liu, M D.; Bayley, H. Catalytic SiteSelective Substrate Processing within a Tubular Nanoreactor. Nat. Nanotech. 2019, 14, 1135-1142

(9) Ying, Y -L.; Long, Y -T. Nanopore-Based Single-Biomolecule Interfaces: from Information to Knowledge. J. Am. Chem. Soc. 2019, 141, 15720-15729.

(10) Xue, L.; Yamazaki, H.; Ren, R.; Wanunu, M.; Ivanov, A P.; Edel, J B. Solid-State Nanopore Sensors. Nat. Rev. Mater. 2020, 5, 931-951.

(11) Galenkamp, N S.; Biesemans, A.; Maglia. G. Directional Conformer Exchange in Dihydrofolate Reductase Revealed by Single-molecule Nanopore Recordings. Nat. Chem. 2020, 12, 481-488.

(12) Tripathi, P.; Benabbas, A.; Mehrafrooz, B.; Yamazaki, H.; Wanunu, M P. Electrical Unfolding of Cytochrome $c$ During Translocation through a Nanopore Constriction..P. Natl. Acad. Sci. USA 2021, 118, 1-10.

(13) Liu, L.; Wu, H -C. DNA-Based Nanopore Sensing. Angew. Chem. Int. Ed. 2016, 55, 15216-15220.

(14) Shin, S.; Luchian, T.; Cheley, S.; And, O B.; Bayley, H. Kinetics of a Reversible Covalent Bond Forming Reaction Observed at the Single-Molecule Level. Angew. Chem. Int. Ed 2002, 114, 3859-3861.

(15) Qiu, K -P.; Fato, T P.; Yuan, B.; Long, Y -T. Toward Precision Measurement and Manipulation of Single-Molecule Reactions by a Confined Space. Small 2019, 15, 1805426-1805420.

(16) Ramsay, W J.; Bell, N A W.; Qing, Y.; Bayley, H. Single-Molecule Observation of the Intermediates in a Catalytic Cycle J. Am. Chem. Soc. 2018, 140, 17538-17546.

(17) Haugland, M M.; Borsley, S.; Cairns-Gibson, D F.; Elmi, A.; Cockroft, S L. Synthetically 
Diversified Protein Nanopores: Resolving Click Reaction Mechanisms. Acs Nano 2019; 13, 4101-4110.

(18) Luchian, T.; Shin, S H.; Bayley, H. Single-Molecule Covalent Chemistry with Spatially Separated Reactants. Angew. Chem. Int. Ed. 2003, 42, 1925-1929.

(19) Pulcu, G S.; Galenkamp, N S.; Qing, Y.; Gasparini, G.; Mikhailova, E.; Matile, S.; Bayley, H. Single-Molecule Kinetics of Growth and Degradation of Cell-Penetrating Poly(disulfide)s. J. Am. Chem. Soc. 2019, 141, 12444-12447.

(20) Qing, Y -J.; Pulcu, G S.; Bell, N A W.; Bayley, H. Bioorthogonal Cycloadditions with Sub-Millisecond Intermediates. Angew. Chem. Int. Ed. 2018, 57, 1218-1221.

(21) Qing, Y -J.; Ionescu, S A.; Pulcu, G S.; Bayley, H. Directional Control of a Processive Molecular Hopper. Science 2018, 361, 908-912.

(22) Steffensen, M B.; Rotem, D.; Bayley, H. Single-Molecule Analysis of Chirality in a Multicomponent Reaction Network. Nat. Chem. 2014, 6, 604-608.

(23) Zhou, B.; Wang, Y.; Cao, C.; Li, D.; Long, Y -T. Monitoring Disulfide Bonds Making and Breaking in Biological Nanopore at Single Molecule Level. Sci. China Chem. 2018, 61, 1385-1388;

(24) Lee, J.; Bayley, H. P. Semisynthetic Protein Nanoreactor for Single-Molecule Chemistry P. Natl. Acad. Sci. USA 2015, 112, 13768-13773.

(25) Bayley, H.; Luchian, T.; Shin, S H.; Steffensen, M B. Single-Molecule Covalent Chemistry in a Protein Nanoreactor, Springer, Heidelberg 2008.

(26) Xie, X.; Lu, H. Single-Molecule Enzymology. J. Bio. Chem. 1999, 274, 15967-15970.

(27) Gu, L.; Braha, O.; Conlan, S.; Cheley, S.; Bayley, H. Stochastic Sensing of Organic Analytes by a Pore-Forming Protein Containing a Molecular Adapter. Nature 1999, 398, 686-690.

(28) Guglielmo M. A Novel Derivation of Collision Theory Rate Constants for a Bimolecular Reaction. J. Math. Chem., 2011. 49, 1544-1557.

(29) Wang, Y -Q.; Cao, C.; Ying, Y -L.; Li, S.; Wang, M -Y.; Huang, J.; Long, Y -T. Rationally Designed Sensing Selectivity and Sensitivity of an Aerolysin Nanopore via Site-Directed Mutagenesis. ACS Sensors 2018, 3, 779-783.

(30) Li, M -Y.; Wang, Y -Q.; Lu, Y.; Ying, Y -L,; Long, Y -T. Single Molecule Study of Hydrogen Bond Interactions between Single Oligonucleotide and Aerolysin Sensing Interface. Front. Chem. 2019, 7, 258.

\section{Author Contributions}

Y.-L. Ying and Y.-T. Long conceived idea. W. Liu. and Z.-L. Yang conducted the experiment and analyzed the data. W. Liu. and C.-N. Yang prepared manuscript. W. Liu, Y.-L. Ying and Y.-T. Long wrote the manuscript.

\section{Acknowledgments}

This research was supported by the National Natural Science Foundation of China (21922405 and 22027806) and the Fundamental Research Funds for the Central Universities (2020102025). Y - L. Ying is sponsored by National Ten Thousand Talent Program for young top-notch talent. We thank Dr. S $-\mathrm{C}$. Liu for data analysis and Dr. $\mathrm{X}-\mathrm{Y}$. Wu for nanopore preparation. 


\section{Competing Financial Interests}

The authors declare no competing financial interest. 